\title{
Relationship between Habitual Physical Activity, Depression and Functional Mobility in Older Adults - A Pilot Cross Sectional Study
}

\author{
Keerthi Mezhunnath*, Haripriya Santhakumar**, Ravish Thunga*** \\ *Assistant Professor, Dept. of Physiotherapy, A.W.H. Special College, Calicut, Kerala - 673003 \\ ** Associate Professor, Laxmi Memorial College of Physiotherapy, Mangalore, 575002 \\ ****Professor and HOD, Department of psychiatry, AJ Institute of Medical Sciences and Research Centre, \\ Mangalore, 575004 \\ Corresponding Author: Keerthi Mezhunnath
}

DOI: https://doi.org/10.52403/ijshr.20220129

\begin{abstract}
Introduction: Ageing is characterized by a decline in mobility function and mental health. Physical activity can possibly play a role in maintaining mental health and functional independence in older adults, but it is seen that elderly persons do not tend to be adequately physically active.

Purpose: To assess physical activity, depression and functional mobility in older adults and to determine the relationship between these factors in them.

Materials and Methods: A pilot cross sectional study was conducted on 40 older adults with age $\geq 65$ years (mean $=69.72, \mathrm{SD}= \pm 4.95$ ). Physical activity was assessed using Short Form International Physical Activity Questionnaire (IPAQ- SF), depression with Short Form Geriatric Depression Scale (GDS- SF) and functional mobility using Time Up and Go test (TUG).

Statistical Analysis: The data was analyzed using Spearman co-efficient of correlation and regression analysis to examine the relationship between IPAQ, GDS and TUG scores.

Results: A significant negative correlation was found between GDS and IPAQ and with IPAQ and TUG. Between GDS and TUG, there was a significant positive correlation. All correlations were significant with $\mathrm{p}$ value $<0.05$. Regression analysis showed that increased IPAQ scores could predict lower TUG scores and lower GDS scores.
\end{abstract}

Conclusion: There was a significant association between level of physical activity, depression, and functional mobility in older adults. Each of these factors needs to be considered when addressing others, considering the relationship between them. Encouraging elderly persons to stay physically active can help maintain their physical and mental health.

Keywords: Older adults, Physical activity, Depression, Functional mobility, Fear of fall, Loneliness.

\section{INTRODUCTION}

Ageing is an inevitable and irreversible phenomenon which has an influence on all systems of the body. Loss of mobility and strength, falls, physical diseases, deterioration in cognition, changes in social status and weakened interpersonal support are some commonly seen changes in aged people. ${ }^{[1]}$ The population of person's aged 60 years or more in India is growing rapidly and it is estimated that the percentage of older adults in India will rise from $8 \%$ in the year 2010 to $19 \%$ in the year 2050. Their number is expected to be 323 million, by mid-century. ${ }^{[2]}$

Aging is characterized by functional decline, and physical activity plays a crucial role in maintaining the functional independence in older adults. Physical activity (PA) is defined as any movement of 
the body produced by skeletal muscles which results in expenditure of energy, and includes all form of activity such as cycling, walking, active play, working out in gym, dancing, competitive sports and work related activity. ${ }^{[3,4]}$ It is associated with a range of health benefits, and its absence can have various harmful effects on health and well being. ${ }^{[5]}$ Regular participation in physical activity may delay, decrease or prevent altogether the ill- effects of several diseases such as heart disease, diabetes and depression. In older adults, it decreases the risk of falling and ensuing functional limitation and also helps to maintain physical and cognitive function. ${ }^{[6]}$ The World Health Organization (WHO) recommends exercise of duration 150 minutes a week for the elderly to derive optimum health benefits, and additional strength and flexibility training at least twice a week. ${ }^{[7]}$

Depression is a mental disorder which is characterized by feelings of sadness, decreased levels of energy and loss of interest in performing activities. A common health problem faced by older adults, it is different from regular mood changes by the extent of its symptoms, the severity and persistence of the disorder. ${ }^{[8]}$ Depression is a common illness all over the world, the prevalence increasing with age, affecting an estimated 350 million people worldwide. It is also related to decreased cognitive, physical and social functioning and to an increased risk of morbidity and also mortality by suicide. Depression can affect the Quality of Life severely and it is being projected to become the third highest cause of disability worldwide by 2020. ${ }^{[9]}$

Deterioration of general health and functional ability is commonly seen in elderly persons. Mobility impairment is one of the foremost causes of decline in function, and is correlated with greater fallrisk among older adults.$^{[10]}$ The aftermath of falls include deconditioning, illness, physical disability, decreased mobility, loss of the ability to perform daily living activities, reduced social participation and burdens on caregivers and society as a whole. [11-14]

The importance of physical activity for independent living in older adults is well understood, but the level of daily activities of elderly persons is seen to decrease with aging. [10] Physical activity has been hypothesized to protect older adults from depression, but health- related habits including physical activity change as a person get older, owing to disease, social changes and environmental alterations. Initiation of physical activity at an advanced age might be difficult because of the decline in physical and mental function. The findings of the Lifestyle Interventions and Independence for Elders (LIFE) study, conducted in the USA, determined that a physical activity programme of moderate intensity, which was structured systematically, was capable of decreasing major and persistent mobility impairment. ${ }^{[15]}$

The association that physical activity, functional mobility and depression have with each other in older adults of India has not been studied with the importance it deserves. It is important to know the factors associated with each of these debilitating aspects of an older person's health, so that healthcare professionals can work on optimal strategies to prevent or rehabilitate them. In this context, a need was identified to study the interrelationship of depression, physical activity and functional mobility. Hence this study was designed in order to assess the relationship between habitual physical activity, depression and functional mobility in older adults.

\section{MATERIALS AND METHODS Research Design and Sample}

This pilot cross sectional study was part of a larger study being conducted with the same objectives. The participants, 20 men and 20 women, were selected from community dwellings and old age homes in Mangaluru. 51 older adults, selected by convenience sampling, who were willing to 
take part in the study were screened for inclusion and exclusion criteria, and if eligible were recruited for the study. Participants of age 65 years and above of both genders, who were functionally independent (able to walk 20 feet without assistance) and those who had a Montreal Cognitive Assessment score greater than or equal to 26 were included in the study.
We excluded participants who were having auditory or visually defects, any neurological disorder such as stroke, Parkinson's disease and multiple sclerosis, those who had undergone amputations, those who reported severe pain while walking and those with lower limb fractures.

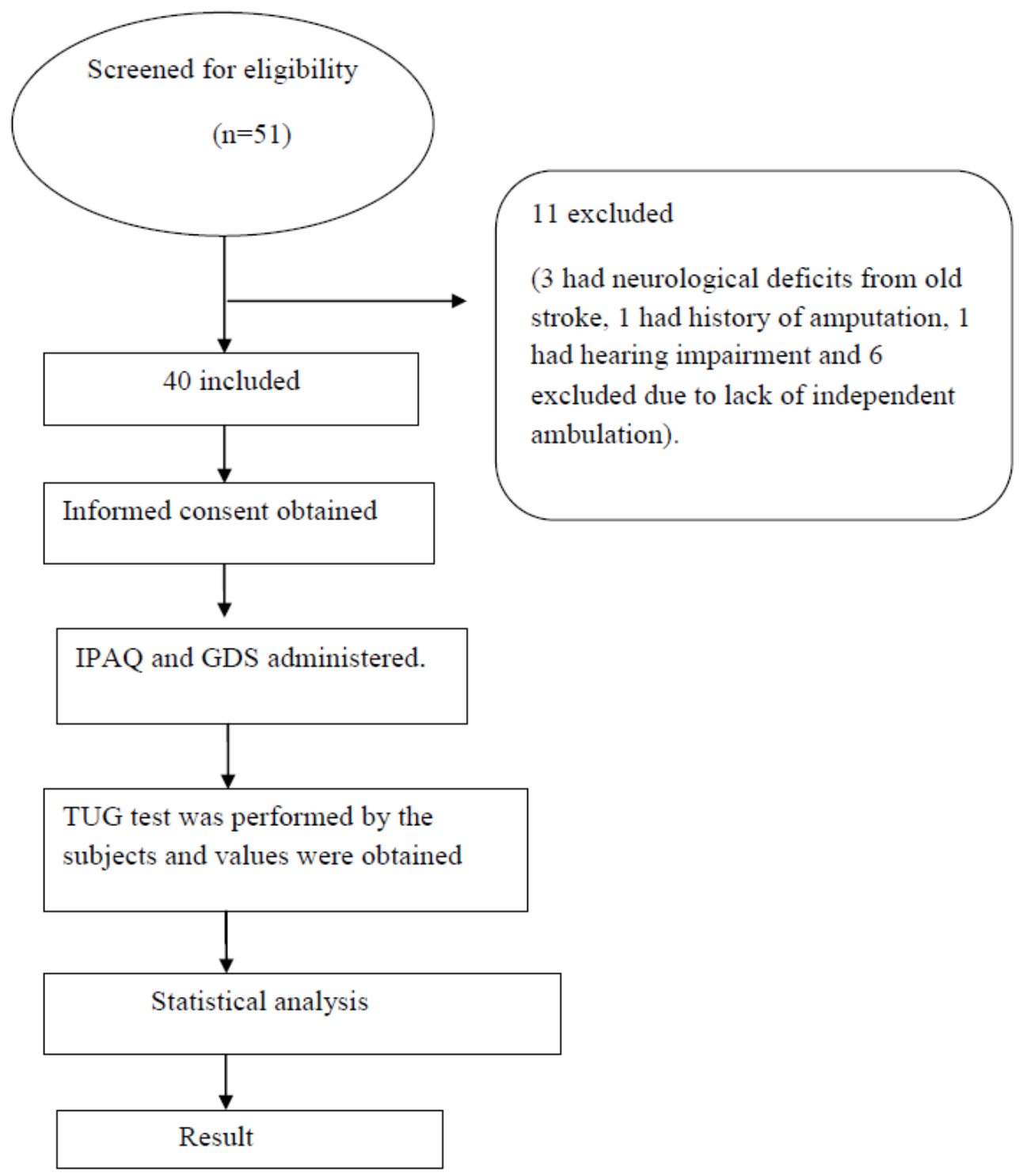

[Table/Fig 1]: Flow diagram of the progress of study

\section{Ethical clearance}

It was obtained from the Ethical Committee of A.J. Institute of Medical Sciences, Mangaluru, South India (Ref no: AJEC/REV/67/2019). The study was carried out in a period of 6 months from October 2019 to March 2020.

\section{Procedure}

The subjects participating in the study were given a patient information sheet containing the study details and informed consent was obtained from them prior to the study. The required demographic details were collected using the demographic data 
collection form. Any doubts about the questionnaire or test were cleared by the investigator. The 3 outcomes were measured using the research instruments on the same day, with a gap of 5 minutes in between the sessions. The scores were recorded on a scoring sheet, and was entered into an excel worksheet on the same day.

\section{Outcome Measures}

Level of physical activity was measured using Short Form International Physical Activity Questionnaire, depression using Short Form Geriatric Depression Scale and functional mobility using Timed Up and Go test. All these scales are available for free use in their unchanged forms. The interview method was used for the questionnaires whenever necessary.

1. The International Physical Activity Questionnaire -Short Form (IPAQSF): This scale was developed in Geneva in 1998 and comprises of a group of 4 questionnaires. The short version comprises of 4 generic items which could e used by either telephone or self-administered, and assesses the time spent doing different physical activities. It takes approximately 10 minutes to administer and records activity at 4 levels of intensity, namely vigorous-intensity activity namely aerobic exercise, moderate-intensity activity like walking and leisure cycling, and sitting. The IPAQ- SF was found to have extensive reliability and validity of about 0.80 and 0.30 respectively. ${ }^{[16]}$

2. Geriatric Depression Scale- Short Form (GDS-SF): The GDS- SF, used to measure symptoms of depression and consisting of 15 questions was developed in 1986 and can be administered in less than 10 minutes. The GDS is appropriate to use in older adults of a wide health spectrum, including healthy, frail and mild to moderately cognitive impaired. It has been used in different community and institution based settings. A sensitivity of $92 \%$ and specificity of $89 \%$ was found when evaluated against several diagnostic criteria. The reliability and validity of the tool have been established in both research and clinical practice settings. ${ }^{[17]}$

\section{Timed Up and Go Test (TUG):} Mobility was measured by the Timed Up and Go (TUG) test. The TUG comprises of basic everyday movements: get up from a chair, walk 3 $\mathrm{m}$, turn around, walk back, and sit down again. A customary walking aid was used if necessary. Intratester and intertester reliability of the TUG test are reported as high, in elderly populations (0.92-0.99). Sensitivity and specificity were both reported to be $87 \% .^{[18]}$

4. Demographic data collection form: A demographic data collection form was used to collect basic information about the participants including age, gender, marital status, education, co morbidities if any and a screening clinical evaluation.

\section{STATISTICAL ANALYSIS}

The collected data was expressed as mean \pm standard deviation (Mean \pm SD), frequency and percentage. Analysis was carried out using Spearman co-efficient of correlation to examine the relationship between IPAQ, GDS and TUG. A p value of $<0.05$ was taken to be significant. A regression analysis was done to analyse how the variables were able to predict one another. The data was analyzed using Microsoft Excel and SPSS version 23.

\section{RESULTS}

Of the 20 men and 20 women who participated in the study, 11 men and 11 women $(55 \%)$ lived in the community, with their spouses or children, and 9 men and 9 women (45\%) lived in homes for the aged. The details of physical activity, depression and functional mobility scores are depicted in [Table/Fig-2]. 
Keerthi Mezhunnath et.al. Relationship between habitual physical activity, depression and functional mobility in older adults - a pilot cross sectional study.

[Table /Fig 2] Descriptive statistics of collected data

\begin{tabular}{|c|c|c|c|}
\hline & Frequency & Percentage & Mean \pm SD \\
\hline \multicolumn{4}{|l|}{ PA level } \\
\hline High & 8 & 20.0 & \\
\hline Low & 12 & 30.0 & $963.600 \pm 723.698$ \\
\hline Moderate & 20 & 50.0 & \\
\hline \multicolumn{4}{|l|}{ Depression } \\
\hline Mild & 12 & 30.0 & \\
\hline Moderate & 11 & 27.5 & $6.100 \pm 2.915$ \\
\hline Normal & 16 & 40.0 & \\
\hline Severe & 1 & 2.5 & \\
\hline \multicolumn{4}{|l|}{ Fall risk } \\
\hline Good mobility & 20 & 50.0 & \\
\hline High fall risk & 12 & 30.0 & $12.910 \pm 3.343$ \\
\hline Normal & 8 & 20.0 & \\
\hline
\end{tabular}

[Table /Fig 3] Correlation between the outcome measures

\begin{tabular}{|l|l|l|}
\hline & r value & p value \\
\hline IPAQ and GDS & -0.889 & 0.000 \\
\hline IPAQ and TUG & -0.908 & 0.000 \\
\hline GDS and TUG & 0.845 & 0.000 \\
\hline
\end{tabular}

There was found to be a significant negative correlation between IPAQ and GDS scores as well as between IPAQ and TUG scores, suggesting that with increased physical activity levels comes lower depression levels and better functional mobility. Between GDS and TUG scores there was a positive correlation, indicating that depressed individuals had lower functional mobility.

[Table / Fig-4] Regression analysis between the outcome measures

\begin{tabular}{|c|c|c|c|c|c|}
\hline \multirow[t]{2}{*}{ DependentVariable: GDS } & \multicolumn{2}{|c|}{ Unstandardized Coefficients } & Standardized Coefficients & \multirow[t]{2}{*}{$\mathbf{T}$} & \multirow[t]{2}{*}{ Sig } \\
\hline & B & Std. Error & Beta & & \\
\hline (Constant) & 9.049 & .494 & & $\begin{array}{c}18.3 \\
21 \\
\end{array}$ & $\begin{array}{l}.00 \\
0\end{array}$ \\
\hline IPAQ & -.003 & .000 & -.709 & $\begin{array}{c}- \\
7.260\end{array}$ & $\begin{array}{c}.00 \\
0\end{array}$ \\
\hline DependentVariable: TUG & \multicolumn{2}{|c|}{ Unstandardized Coefficients } & Standardized Coefficients & \multirow[t]{2}{*}{$\mathrm{T}$} & \multirow[t]{2}{*}{ Sig } \\
\hline & $\mathrm{B}$ & Std. Error & Beta & & \\
\hline (Constant) & 16.733 & .568 & \multirow[t]{2}{*}{-.733} & $\begin{array}{l}29.4 \\
42 \\
\end{array}$ & $\begin{array}{c}.00 \\
0 \\
\end{array}$ \\
\hline IPAQ & -.004 & .000 & & $\begin{array}{c}- \\
7.781\end{array}$ & $\begin{array}{c}.00 \\
0\end{array}$ \\
\hline
\end{tabular}

The coefficient table of regression analysis shows that IPAQ contributes statistically significantly to the model. The regression equation given by the model is, GDS $=0.9049-0.003$ (IPAQ). B value -0.003 which indicates that, for every unit increase in IPAQ, there is -0.003 unit decrease in the predicted GDS score. Similarly, the regression equation given by the model for TUG is TUG $=16.733-0.004$ (IPAQ). Here $B$ value is -0.004 which indicates that for every unit increase in IPAQ, there is a 0.004 unit decrease in the predicted value of TUG score. These values indicate that higher levels of physical activity are able to predict lower depression levels and higher functional mobility levels.

\section{DISCUSSION}

Considering the rapidly expanding numbers of older adults worldwide, and especially in India $(7.7 \%$ of the total population in India are older adults), interventions to smoothen over the aging process and to maintain functional ability and independence in older adults are gaining importance. ${ }^{[19]}$

The present study utilized a cross sectional study design with convenience sampling technique to determine the correlation between physical activity assessed by IPAQ- SF, depression by GDSSF and functional mobility by TUG in 40 older men and women, all of which are parameters that are important in maintaining and improving function and quality of life in older adults. The participants were of the age range 65 to 88 , with the mean age of 69.725 \pm 4.956 . Correlation analysis showed that all coefficients were significant with $\mathrm{p}$ value $<0.05$. A significant negative correlation was found between GDS and IPAQ and with IPAQ and TUG, which affirms that, as the physical activity increases, the chances for the occurrence of depression decrease, and functional mobility increases.

This study observed a negative association between depressive symptoms and physical activity levels in elderly 
people. Some other authors like Yoshida in 2015, Hallegren in 2019 and Rebar in 2015 have also found that being physically active and maintaining physical activity could decrease the incidence and severity of depressive symptoms. ${ }^{[20-22]}$ Depression has been seen to decrease hippocampal volumes, lessen levels of neurogenesis markers and increase levels of oxidant and inflammatory markers. These abnormal physiological changes can be modulated and possibly reversed with physical activity, which may explain to some extent why physically active people suffer fewer depressive symptoms. Regular exercise may also help the individuals to distract from their daily worries and promote self-esteem or perceptions of physical competence. [21] The Physical Activity Guidelines Advisory Committee, 2008, also has stated that people who are regularly physically active have significantly lower odds of an onset of clinical depression symptoms and of clinical anxiety symptoms. ${ }^{[22]}$

The results add to the available evidence about how increasing the quantum of physical activity in non- clinical populations can decrease levels of depression and prevent the onset of clinical depression.

The present study also found a negative correlation between depression levels and functional mobility, similar results to which were found by Mishra in 2017 and Sanchez- Martinez in 2016. A vicious cycle relationship was also seen between depression and physical disability, with depression leading to physicaldisability and that leading to further depressive symptoms. The progression of mobility restriction into disability was seen to be accelerated by depression. ${ }^{[23]}$ The exact mechanisms which cause the association between mobility and depression are yet to be understood fully, but it is clear that the two have some mediators in common, such as poor health status and deterioration in physical function. Any clinician, whether treating depression or treating restrictions in mobility cannot afford to underrate the impact that depressive mood has on mobility and interventions to improve it.

Physical activity levels and functional mobility levels displayed a positive correlation; this correlation is logical since physical activity comprises of mobility related activities, and an increase in one is bound to affect the other positively. Marco Pahor et al in 2014 had found a moderately intense physical activity program to be effective in decreasing major mobility disability long term among older adults at risk of disability. ${ }^{[24]}$ People of older age groups experience a decline in functional mobility, which is a result of a gradual and escalating reduction in aerobic endurance, postural balance and muscle performance which in turn decreases their functional ability and independence, further reducing the level of physical activity. ${ }^{[25]}$

The most commonly reported types of physical activity in this study were walking, housework, gardening and taking care of grandchildren. Organized physical activity was at a bare minimum, except for walking in a safe and controlled environment. Considering the cultural fabric of India, physical activity, including activities of daily living, is not a priority for elderly. Those living in the rural regions are seen to engage in some physical activity related to their lifestyle, but in the urban dwelling elderly, the trend of low physical activity is mostly seen. Most elderly persons live with their children or have domestic help at home, and the common understanding is that exercise is dangerous and rest is the best medicine for the elderly. A very important gap in the knowledge of the common man related to the benefits of physical exercise is the connection between physical exercise and mental health. The literature provides a good quantum of evidence on the benefit of exercise on cognition, memory and to a lesser extent, depression. ${ }^{[26-29]}$ The findings of the present study can be used as an addition to the knowledge in the field, and used for forming the basis of education programs for the elderly and for caregivers. 
The lower incidence of depressive symptoms experienced by the physically active and mobile individuals is indicative of the protective effect that physical activity has on depression. Regression analysis also showed that mobility problems and low levels of physical activity are able to predict depressive symptoms in the elderly. It follows that interventions and education programs aimed at improving mobility and levels of physical activity can play a role in improving the mental health and well being of older adults. Also, physical activity programs can delay the onset of age- related mobility impairments, thus reducing the odds of the elderly developing depressive symptoms. That said, initiation of physical activity in persons of advanced years might be difficult, due to mental, physical and cognitive constraints, in addition to societal restraints and fear of injury. Hence it is recommended that individuals initiate physical activity when younger, at least during middle age, in order to maintain their mental and physical health during old age.

\section{Limitation}

This study is with a small number of limitations. First, as this is a pilot cross sectional study, the ability to establish a cause- and- effect relationship between the variables is limited. Secondly, being a pilot study, the analysis was carried out on a limited sample size. The third limitation is the reliance on self- reported data for PA and depression, though we used previously developed reliable and valid tools. Due to these limitations, the results of this study require further investigation to be conducted for establishing a cause and effect relationship between the variables and a higher reliability of the correlations found.

There were no adverse effects noted during conduct of the study, though some participants found the procedure of answering questions to be tedious, and others were hesitant to give information about their mental status.

\section{CONCLUSION}

Regular physical activity could be a simple and valuable way to prevent depression and to improve functional mobility in older adults. Initiating interventions and education programs which integrate physical activity into the daily lives of the elderly can prevent depression, reduce incidence of falls and provide greater functional independence.

\section{Conflict of Interest}

The authors agreed that there was no source of conflict of interest.

\section{ACKNOWLEDGEMENT}

The authors acknowledge the contribution of Mrs. Reshma Kolar, who conducted the statistical analysis required for the study.

\section{Source of Funding: None}

\section{Ethical Approval: Approved}

\section{REFERENCES}

1. Polat Ü, Kahraman B, Kaynak İ, Görgülü Ü. Relationship among health-related quality of life, depression and awareness of home care services in elderly patients.GeriatrGerontol Int. 2016;16(11):1211-9.

2. Fried LP, Bush TL. Morbidity as a focus of preventive health care in the elderly. Epidemiologic Reviews. 1988; 10:48-64.

3. Pantelić S, Ranđelović N, Milanović Z, Trajković N, Sporiš G, Kostić R. Physical activity of elderly women in terms of age. FactaUniversitatis. Series: PhysEduc Sport. 2012; 10(4):289-96.

4. Van Lummel RC, Walgaard S, Pijnappels M, Elders PJ, Garcia-Aymerich J, van DieënJH, et al. Physical performance and physical activity in older adults: associated but separate domains of physical function in old age. PloS one. 2015; 10(12):e0144048.

5. Ströhle A. Physical activity, exercise, depression and anxiety disorders. J Neural Transm.2009; 116(6):777.

6. Smith GL, Banting L, Eime R, O'Sullivan G, Van Uffelen JG. The association between social support and physical activity in older 
adults: a systematic review. Int J BehavNutr Phys. 2017 1; 14(1):56.

7. WHO, 2010. Global Recommendations on Physical Activity for Health. World Health Organization, Geneva.

8. Park M, Reynolds CF. Depression among older adults with diabetes mellitus. ClinGeriatr Med. 2015 1;31(1):117-37

9. Lindwall M, Larsman P, Hagger MS. The reciprocal relationship between physical activity and depression in older European adults: A prospective cross-lagged panel design using SHARE data. Health Psychol. 2011; 30(4):453.

10. Lin SI, Lee HC, Chang KC, Yang YC, TsauoJY. Functional mobility and its contributing factors for older adults in different cities in Taiwan. J Formos Med Assoc . 2017 1; 116(2):72-9.

11. Mishra N, Mishra AK, Bidija M. A study on correlation between depression, fear of fall and quality of life in elderly individuals. Int J Res Med Sci.Int J Res Med Sci. 2017; 5:1456-60.

12. Auais M, Alvarado B, Guerra R, Curcio C, Freeman EE, Ylli A, Guralnik J, Deshpande $\mathrm{N}$. Fear of falling and its association with life-space mobility of older adults: a crosssectional analysis using data from five international sites. Age and ageing. 2017; 46(3):459-65.

13. Viljanen A., Kulmala J., Rantakokko M., Koskenvuo M., Kaprio J., Rantanen T. Fear of Falling and Coexisting Sensory Difficulties As Predictors of Mobility Decline in Older Women. J GerontolSer A: BiolSci Med Sci 2012; 67 (11):1230-7.

14. Moreira Bde S, Dos AnjosDM, Pereira DS, SampaioRF, Pereira LS, Dias RC.

15. Kirkwood RN. The geriatric depression scale and the timed up and go test predict fear of falling in community-dwelling elderly women with type 2 diabetes mellitus: a cross-sectional study. BMC Geriatr. 2016; 16(1):56.

16. Fielding RA, GuralnikJM, King AC, Pahor M, McDermott MM, Tudor-Locke C, et al. Dose of physical activity, physical functioning and disability risk in mobilitylimited older adults: Results from the LIFE study randomized trial. PloS one. 2017; 12(8): 0182155.

17. Craig CL, Marshall AL, Sjöström M, Bauman AE, Booth ML, Ainsworth BE, Pratt M, Ekelund UL, Yngve A, SallisJF,
Oja P. International physical activity questionnaire: 12- country reliability and validity. Medicine \& science in sports \& exercise. 2003; 35(8):1381- 95.

18. Nyunt MS, Fones C, Niti M, Ng TP. Criterion-based validity and reliability of the Geriatric Depression Screening Scale (GDS-15) in a large validation sample of community-living Asian older adults. Aging MentHealth.2009; 13(3):376-82.

19. Shumway- Cook A, Brauer S, Woolacott M. Predicting the probability for falls in communty- dwelling older adults using the Timed Up and Go Test. PhysTher. 2000; 80(9): 896- 903.

20. Amarya S, Singh K, Sabharwal M. Changes during aging and their association with malnutrition. J.Gerontol. Geriatr.2015; 6(3):78-84.

21. Yoshida Y, Iwasa H, Kumagai S, Suzuki T, Awata S, Yoshida H. Longitudinal association between habitual physical activity and depressive symptoms in older people. Psychiatry ClinNeurosci2015; 69(11): 686-92.

22. Hallgren M, Lundin A, Vancampfort D, Stubbs B, Schuch F, Bellocco R, et al. Prospective associations between physical activity and clinician diagnosed major depressive disorder in adults: a 13-year cohort study. Prev Med .2019; 118:38-43

23. Rebar AL, Stanton R, Geard D, Short C, Duncan MJ, Vandelanotte C. A meta-metaanalysis of the effect of physical activity on depression and anxiety in non-clinical adult populations. Health Psychol Rev. 2015; 9(3):366-78.

24. Sánchez-Martínez M, Castell MV, González-MontalvoJI, De la Cruz JJ, Banegas JR, Otero Á. Transitions in functional status of community dwelling older adults: Impact of physical performance, depression and cognition. EurGeriatr Med. 2016; 7(2):111-6.

25. Pahor M, GuralnikJM, AmbrosiusWT, Blair S, Bonds DE, Church TS, EspelandMA, et al.Effect of structured physical activity on prevention of major mobility disability in older adults: the LIFE study randomized clinical trial. JAMA. 2014; 311(23):2387-96.

26. Andrade daSilva R, SáBrandão G, Silva AS, UrbanoJJ, Oliveira EF, Oliveira LV, Camelier AA, SáBrandão G. Physical Activity Level, Functional Mobility and Fall 
Keerthi Mezhunnath et.al. Relationship between habitual physical activity, depression and functional mobility in older adults - a pilot cross sectional study.

Risk in the Elderly. Manual Therapy, Posturology\& Rehabilitation Journal= Revista Manual Therapy. 2017; 15.

27. Lampinen P, Heikkinen E. Reduced mobility and physical activity as predictors of depressive symptoms among communitydwelling older adults: an eight-year followup study. Aging ClinExp Res. 2003; 15(3): 205-11.

28. Lü J, Fu W, Liu Y. Physical activity and cognitive function among older adults in China: a systematic review. J Sport Health Sci. 2016; 5(3):287-96.

29. McEwen SC, Siddarth P, Abedelsater B, Kim Y, Mui W, Wu P, Emerson ND, Lee J, Greenberg S, Shelton T, Kaiser S. Simultaneous aerobic exercise and memory training program in older adults with subjective memory impairments. J Alzheimer's Dis. 2018; 62(2):795-806.

30. Schuch FB, Vancampfort D, Rosenbaum S, Richards J, Ward PB, Veronese N, Solmi M, Cadore EL, Stubbs B. Exercise for depression in older adults: a meta-analysis of randomized controlled trials adjusting for publication bias. Braz J Psychiatry. 2016; 38(3):247-54.

How to cite this article: Keerthi Mezhunnath, Haripriya Santhakumar, Ravish Thunga. Relationship between habitual physical activity, depression and functional mobility in older adults - a pilot cross sectional study. International Journal of Science \& Healthcare Research. 2022; 7(1): 179-187. DOI: https:// doi.org/10.52403/ijshr.20220129 